\title{
Cooperative Linear Precoding for Multi-User MISO Visible Light Communications
}

\author{
Roser Viñals, Olga Muñoz, Adrián Agustín, and Josep Vidal \\ Dept. Signal Theory and Communications - Universitat Politècnica de Catalunya (UPC), Barcelona, Spain \\ Emails: roser.vinals@alu-etsetb.upc.edu, \{olga.munoz, adrian.agustin, josep.vidal\}@upc.edu
}

\begin{abstract}
This paper develops and evaluates precoding techniques for coordinated joint transmission in visible light communications (VLC). A multi-user multiple-input single-output (MISO) setup is adopted. Transmitters are equipped with light emitting diode (LED) arrays, while receivers incorporate a single photodetector (PD). We design zero forcing (ZF) linear precoders considering, as figure of merit, the weighted sumrate for multilevel pulse amplitude modulation (PAM) and take into account the optical power constraints. We present two approaches. First, we formulate the design as a convex optimization problem and find its exact solution. Second, by considering certain approximations, we find an alternative precoder that despite being suboptimal can be computed in an almost closed-form, achieving a good trade-off between performance and complexity. Finally, we demonstrate that the proposed approaches largely outperform the conventional pseudo-inverse precoding and single-user TDMA approaches.
\end{abstract}

Index Terms-Visible light communications, Multi-user multiple-input single-output, Adaptive modulation, Zero forcing, Weighted sum-rate maximization.

\section{INTRODUCTION}

Lighting is going through revolution. The use of light emitting diodes (LEDs) has greatly increased during the last few years, meanwhile incandescent and fluorescent lamps have become obsolete. LEDs, apart from being extremely highly energy efficient, are able to switch to different light intensities at very high speed. This fact, along with the looming crisis of the radio frequency (RF) spectrum, has paved the way for the growing interest in visible light communication (VLC) that has led to significant advances in the last years. VLC systems use the huge license-free spectrum of approximately $400 \mathrm{THz}$, offering an interesting alternative to $\mathrm{RF}$ for wireless communication, while making use of existing lighting infrastructure [1].

Usually, several LEDs are employed in a light installation to provide enough illumination and this can be exploited to create optical multiple-input single-output (MISO) communications if single photodetectors (PD) are implemented at the receiver. The coordination of transmissions for the downlink, i.e. from multiple lamps towards multiple users, can be done through linear precoding. Particularly, zero-forcing (ZF) is a simple linear precoding method that decouples the

The research leading to these results has received funding from the Spanish Ministry of Economy and Competitiveness (Ministerio de Economía y Competitividad) through the project TEC2016-77148-C2-1-R (RUNNER) and from the Catalan Government (AGAUR) through the grant 2014 SGR 60 multi-user channel into multiple independent subchannels [2]. Nevertheless, this precoding technique can not be directly applied in VLC systems due to the different nature of the visible light channel.

In RF systems, modulation techniques include amplitude, phase, and frequency modulation. The antenna output in such systems is an electrical signal that is proportional to the amplitude of the electrical field. This stands in contrast with VLC, where the signal modulates only the amplitude of the light. These modulations are called intensitymodulated/direct-detection (IM/DD) modulations and imply critical differences between VLC and RF [3]. The two main differences can be summarized to:

1) The waveform transmitted, i.e. the channel input, in visible light represents instantaneous optical power measured in watts and needs to be real-valued and nonnegative (non-negativity constraint).

2) The power constraints are directly applied on the amplitude of the waveform transmitted. In this work, we are going to limit the maximum instantaneous optical power emitted by each LED (maximum optical power constraint).

There have been several studies on multi-user multiple-input single-output (MU-MISO) for VLC. In [4], a Block Diagonalization system for VLC has been studied without taking into consideration the positivity constraint. A capacity bound of the VLC channel is considered in [5] and [6] for the precoder design, although it is not described how this capacity bound can be achieved in practice. Moreover, the MU-MISO precoder designed in [6] for indoor VLC systems takes into account the non-negativity constraint but not the maximum optical power constraint. Once the problem is formulated, the authors adopt alternative tighter constraints to be able to solve the problem. As a result, the designed precoder achieves only a lower bound of the maximum achievable sumrate. Differently, in [7] the achievable rate is derived from the expression of the bit error rate (BER). Nevertheless, the structure of the precoding matrix is fixed beforehand, being therefore suboptimal in terms of sum-rate.

In this work, we carry out the design of ZF precoders for VLC systems considering both the non-negativity constraint and the maximum optical power constraint per-LED. As demonstrated in [8], for RF communications, the pseudoinverse precoder is optimal among the generalized inverses when a total power constraint is imposed. However, when considering per-LED power constraints, other schemes out- 
perform this precoder as it will be shown later. The design of multiple-input multiple-output (MIMO) schemes with individual per-antenna constraints is considered in [8], [9] for a broadcast RF wireless channel. However, these results can not be directly applied in VLC due to the different nature of the channel.

To address the design of ZF schemes suitable to VLC systems, we proceed with the design of ZF precoders through the formulation of an optimization problem. The goal is to maximize the weighted sum-rate of the users subject to both the non-negativity constraint and the maximum optical power constraint per-LED for multilevel pulse amplitude modulation (MPAM) modulations. To solve this nontrivial problem we will resort to convex optimization techniques and we will first find the exact solution to the problem. Then, we will find a suboptimal almost closed-form solution to the problem. The benefit in this case is the lower complexity to compute the precoders of the users.

Our contributions are twofold:

- On the one hand, we provide the mechanism to compute the ZF MU-MISO scheme that is optimum in terms of sum-rate for the VLC channel, while only suboptimal approaches had been provided so far in the literature.

- On the other hand, we obtain a suboptimal (in terms of sum-rate) ZF MU-MISO scheme that can be computed more easily. Although this suboptimal scheme shares some ideas with the scheme in [6], our approach achieves a highest lower bound of the achievable sum-rate as we will be explaining in Section III-B.

The rest of the paper is organized as follows. Section II introduces the system model for the downlink transmission in a multi-user VLC system, and the non-negative and maximum power constraints are properly formulated. Section III is the core of this paper, addressing the design of the $\mathrm{ZF}$ precoders for VLC systems proposed in this paper. Section IV provides numerical results on the performance of the proposed schemes. Finally, Section V concludes the paper.

The following notation is used. Bold upper case letters denote matrices, bold lower case letters denote column vectors, and standard lower case letters denote scalars. The superscripts $(\cdot)^{T},(\cdot)^{-1}$ and $(\cdot)^{\dagger}$ denote the transpose, matrix inverse and pseudo-inverse, respectively. The operators $\operatorname{Tr}(\cdot), \mathbb{E}[\cdot],\|\cdot\|_{p}$ and $\operatorname{diag}(\cdot)$ denote the trace, the statistical expectation, the p-norm and a diagonal matrix, respectively. Finally, the matrix $\mathbf{I}$ denotes the identity matrix and $\mathbf{X} \succeq 0$ means that $\mathbf{X}$ is positive semidefinite.

\section{SYSTEM MODEL}

We consider a MU-MISO VLC system consisting of $L$ LEDs and $K$ users equipped with a single PD. The discretetime baseband signal of the MISO multi-user broadcast channel is given by

$$
y_{k}=\gamma \mathbf{h}_{k}^{T} \mathbf{x}+n_{k}, \quad k=1, \ldots, K
$$

where $\mathbf{x} \in \mathbb{R}^{L \mathrm{x} 1}$ are the optical transmitted samples and $y_{k}$ the electrical signal samples received by the $k$-th user; $\mathbf{h}_{k} \in \mathbb{R}^{L \times 1}$ denotes the downlink channel from all the LEDs to the $k$ th user; $\gamma$ is the responsivity ${ }^{1}$; and $n_{k}$ denotes the receiver real valued additive white Gaussian noise with zero mean and variance $\sigma_{k}^{2}$ given by the contributions of the thermal and shot noise [10].

The shot noise variance can be expressed as

$$
\sigma_{\text {shot }}^{2}=2 q \gamma P_{r} B+2 q I_{B} I_{2} B
$$

where $q$ is the electronic charge, $P_{r}$ is the received optical power, $B$ is the equivalent noise bandwidth of the PD, $I_{B}$ is the photocurrent due to background radiation and $I_{2}$ is a noise bandwidth factor. On the other hand, the thermal-noise variance is given by

$$
\sigma_{\text {thermal }}^{2}=\frac{8 \pi k_{B} T_{k}}{G_{o l}} C_{p d} A I_{2} B^{2}+\frac{16 \pi^{2} k_{B} T_{k} \eta}{g_{m}} C_{p d}^{2} A_{r}^{2} I_{3} B^{3}
$$

where $k_{B}$ is the Boltzmann's constant, $T_{k}$ is the absolute temperature, $G_{o l}$ is the open-loop voltage gain, $C_{p d}$ is the capacitance of the photodetector per unit area, $\eta$ is the field effect transistor (FET) channel noise, $g_{m}$ is the FET transconductance and $I_{2}$ is another noise bandwidth factor.

The Direct Current (DC) channel gain between the $l$-th LED and the $k$-th PD is given by [11], [12]

$$
h_{l, k}= \begin{cases}\frac{(n+1) A_{r}}{2 \pi D_{l, k}^{2}} \cos ^{n}(\beta) \cos (\alpha) T_{s}(\alpha) g(\alpha) & 0 \leq \alpha \leq F O V \\ 0 & \alpha>F O V\end{cases}
$$

where $A_{r}$ is the effective receiver's area, $n$ is the order of the Lambertian source given by $n=\frac{\ln 2}{\ln \left(\cos \Phi_{1 / 2}\right)}$ where $\Phi_{1 / 2}$ is the semi-angle at half-power, $\alpha$ and $\beta$ are the incident angle and irradiation angle, respectively, and $D_{l, k}$ the distance between transmitter $l$-th and receiver $k$-th. $T_{s}(\alpha)$ is the gain of the optical filter and $g(\alpha)$ the gain of the optical concentrator that, for a Lambertian source, is given by

$$
g(\alpha)= \begin{cases}\frac{\kappa^{2}}{\sin ^{2}(F O V)} & 0 \leq \alpha \leq F O V \\ 0 & \alpha>F O V\end{cases}
$$

with $\kappa$ representing the refractive index of the concentrator. We assume that full knowledge of channel state information (CSI) is available at the transmitter and receiver.

Gathering the expressions in equation (1) for the $K$ users, we can equivalently write

$$
\mathbf{y}=\gamma \mathbf{H} \mathbf{x}+\mathbf{n}
$$

where $\mathbf{y} \in \mathbb{R}^{K \times 1}, \mathbf{H}=\left[\mathbf{h}_{1}, \mathbf{h}_{2}, \ldots, \mathbf{h}_{K}\right]^{T} \in \mathbb{R}^{K \times L}$ and $\mathbf{n} \in$ $\mathbb{R}^{K \times 1}$. For convenience, we assume $L \geq K$ so that $\mathbf{H}$ is full row-rank. Without loss of generality, we can further express $\mathbf{x}$ as

$$
\mathbf{x}=\mathbf{W} \mathbf{s}+\mathbf{b}
$$

where $\mathbf{W}=\left[\mathbf{w}_{1}, \ldots, \mathbf{w}_{K}\right] \in \mathbb{R}^{L \times K}$ denotes the precoding matrix and $\mathbf{s} \in \mathbb{R}^{K \times 1}$ is the information-bearing signal. In Figure 1, the system diagram is shown.

\footnotetext{
${ }^{1}$ The responsivity $\gamma[\mathrm{A} / \mathrm{W}]$ is the ratio of the output photocurrent to the input optical power and characterizes the optical-to-electrical $(\mathrm{O} / \mathrm{E})$ conversion
} 


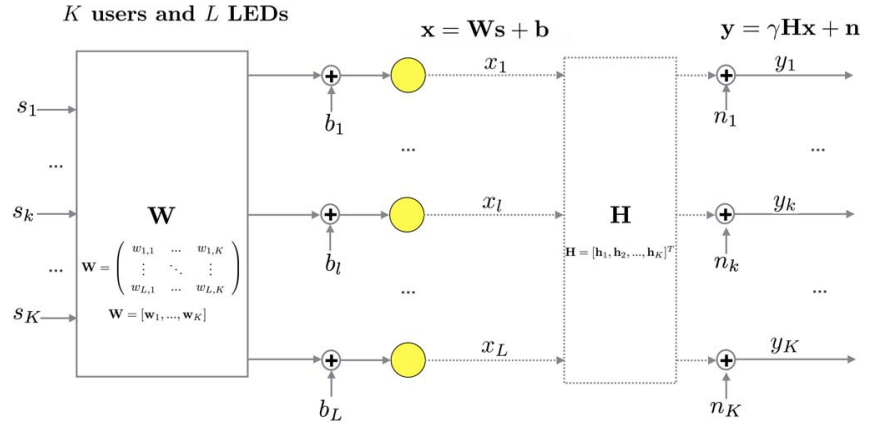

Figure 1. MU-MISO VLC system diagram.

It is assumed that the components of $\mathbf{s}$ are selected from a MPAM constellation and are normalized to the range of $[-1,1]$. Since the VLC systems use IM/DD, a bias $\mathbf{b}=$ $\left[b_{1}, \ldots, b_{L}\right]^{T} \in \mathbb{R}^{L \times 1}$ should be added in order to fulfill the non-negativity requirement at every LED. This constraint can be expressed as

$$
x_{l}=\sum_{k=1}^{K} w_{l, k} s_{k}+b_{l} \geq 0, \quad l=1, \ldots, L .
$$

Taking the worst case, i.e. when the right size of the inequality is minimal $\left(w_{l, k} s_{k}=-\left|w_{l, k}\right| \forall k, l\right)$, the precoding weights have to be chosen in such a way that

$$
\begin{gathered}
\text { Non - negativity } \\
\text { constraint }
\end{gathered}: \sum_{k=1}^{K}\left|w_{l, k}\right| \leq b_{l}, \quad \forall l
$$

On the other hand, unlike RF transmitters which are constrained in terms of electrical power, each LED is constrained in terms of optical power, as the instantaneous transmitted optical power must ensure that:

$$
x_{l}=\sum_{k=1}^{K} w_{l, k} s_{k}+b_{l} \leq P_{\text {opt }_{\max }}, \quad l=1, \ldots, L
$$

where $P_{o p t_{\max },}$ is the maximum instantaneous optical power for the $l$-th LED. Without loss of generality, we assume that it is the same for all the LEDs. Again, taking the worst case that is given when $w_{l, k} s_{k}=\left|w_{l, k}\right|$, the inequality (10) leads to

$$
\begin{gathered}
\text { Optical power } \\
\text { constraint }
\end{gathered}: \sum_{k=1}^{K}\left|w_{l, k}\right| \leq P_{o p t_{\max }}-b_{l}, \quad \forall l
$$

By combining (6) and (7), we obtain

$$
\mathbf{y}=\gamma(\mathbf{H W} \mathbf{s}+\mathbf{H b})+\mathbf{n}
$$

where $\mathbf{H b}$ is the so-called direct current (DC) component that can be easily estimated and removed by the receiver.

After removing the DC component, the electrical signal-tonoise ratio (SNR) of the $k$-th user can be expressed as

$$
\mathrm{SNR}=\frac{\gamma^{2}\left|\mathbf{h}_{k}^{T} \mathbf{w}_{k}\right|^{2} P_{s, \text { elec }}}{\sigma_{k}^{2}}
$$

where $P_{s, e l e c}$ is the electrical modulation power given by

$$
P_{s, \text { elec }}=\mathbb{E}\left\{s^{2}\right\}=\frac{1}{3} \frac{(M+1)}{(M-1)} .
$$

with $M$ indicating the number of available amplitude levels, i.e. the number of symbols of the MPAM constellation ${ }^{2}$.

From [13], the BER expression for a MPAM constellation can be approximated using the Chernoff upper bound leading to

$$
\mathrm{BER} \leq 0.1 \exp \left[-\frac{1.5 \mathrm{SNR}}{M^{2}-1}\right]
$$

that combined with (13) gives rise the following BER bound:

$$
\mathrm{BER} \leq 0.1 \exp \left[-\frac{1.5 \gamma^{2}\left|\mathbf{h}_{k}^{T} \mathbf{w}_{k}\right|^{2} P_{s, \text { elec }}}{\sigma_{k}^{2}\left(M^{2}-1\right)}\right] .
$$

\section{ZERO FORCING PRECODING}

In this section, firstly, we consider the design of the ZF precoder optimal in terms of maximum weighted sum-rate, considering both the positivity constraint and the maximum optical power constraint per-LED. Then, we consider an approximation of the cost function and obtain an alternative problem for which an almost closed-solution can be found. Finally, we obtain the ZF precoder with the pseudo-inverse for benchmarking purposes.

\section{A. Optimal weighted sum-rate zero forcing precoding}

Let us turn our attention to the BER upper-bound from (16). Substituting the modulation power expression (14), the BER bound can be further expressed as

$$
\operatorname{BER}_{\mathrm{MPAM}}(\mathrm{SNR}) \leq 0.1 \exp \left[-\frac{\gamma^{2}\left|\mathbf{h}_{k}^{T} \mathbf{w}_{k}\right|^{2}}{2 \sigma_{k}^{2}(M-1)^{2}}\right] .
$$

Since $M=2^{r}$, (17) leads to the following rate lower-bound:

$$
r \geq \log _{2}\left(1+c_{o}\left|\mathbf{h}_{k}^{T} \mathbf{w}_{k}\right|\right)
$$

where $c_{o}=\frac{\gamma}{\sigma \sqrt{2 \log \left(\frac{0.1}{\mathrm{BER}_{\mathrm{MPAM}}}\right)}}$.

Remark 1 . The noise variance is the sum of contributions from thermal and shot noise. The shot noise depends on the optical power received, whereas the thermal noise is considered independent. For convenience, we have assumed that the shot noise variance is independent of the power received and thus, is the same for all the users:

$$
\sigma_{\text {shot }}^{2}=2 q \gamma P_{r} B+2 q I_{B} I_{2} B \approx 2 q I_{B} I_{2} B .
$$

The precoding matrix should satisfy the non-negativity and optical power constraints. Note that the constraints (9) and (11) can be expressed as a single constraint

$$
\sum_{k=1}^{K}\left|w_{l, k}\right| \leq P_{l}, \quad l=1, \ldots, L
$$

where $P_{l}=\min \left(b_{l}, P_{o p t_{\max }}-b_{l}\right)$. Note that this constraint is enlarged when $b_{l}=\frac{P_{o p t} t_{\max }}{2} \forall l$ and, consequently, $P_{l}$ is a known constant that can be denoted by $P=\frac{P_{o p t_{\max }}}{2}$. Taking the optimum value of $b_{l}$, i.e $b_{l}^{*}=\frac{P_{o p t_{\max }}}{2} \forall l$, we

\footnotetext{
${ }^{2}$ The optical power is converted to current in the receiver, according to the responsivity factor $\gamma$ (already considered in the model). Thus, the receiver electrical SNR in VLC is proportional to the square of the received optical average power while in $\mathrm{RF}$ is directly proportional to the received average power.
} 
can rewrite this constraint as $\sum_{k=1}^{K}\left|\boldsymbol{j}_{l}^{T} \mathbf{w}_{k}\right| \leq P$ where $j_{l} \triangleq(0, \ldots, 0,1,0, \ldots, 0)^{T}$ contains a 1 in the $l$-th position, while the rest of elements are zero.

In addition to previous constraints, we consider the ZF precoding scheme which eliminates the inter-user interference, i.e. $\mathbf{h}_{j}^{T} \mathbf{w}_{k}=0, \forall j \neq k$.

We are now ready to formulate the weighted sum-rate maximization problem with the ZF precoding and per-LED power constraints as follows:

$$
\begin{array}{cl}
\text { (PA1): } \underset{\left\{\mathbf{w}_{k}\right\}}{\operatorname{maximize}} & \sum_{k=1}^{K} u_{k} \log _{2}\left(1+c_{o}\left|\mathbf{h}_{k}^{T} \mathbf{w}_{k}\right|\right) \\
\text { s.t. } & \mathbf{h}_{j}^{T} \mathbf{w}_{k}=0, \forall j \neq k \\
& \sum_{k=1}^{K}\left|\boldsymbol{j}_{l}^{T} \mathbf{w}_{k}\right| \leq P, \forall l
\end{array}
$$

where $u_{k}$ is the rate weight for the $k$-th user.

The previous problem is not a concave problem because of the absolute value within the logarithm of the cost function. However, note that we can add the constraint $\mathbf{h}_{k}^{T} \mathbf{w}_{k} \geq 0 \forall k$, without loss of optimality. This can be easily explained as follows. If the optimal solution of (PB1) were $\mathbf{w}_{k}$ such that $\mathbf{h}_{k}^{T} \mathbf{w}_{k} \leq 0$, we could change the sign of $\mathbf{w}_{k}$, i.e. $\widetilde{\mathbf{w}}_{k}=-\mathbf{w}_{k}$ , in order to satisfy $\mathbf{h}_{k}^{T} \widetilde{\mathbf{w}}_{k} \geq 0$. After changing the weights sign, the restrictions would still be met and the objective function would not be penalized. With this new restriction we can change the objective function leading to a concave problem.

Thus, we rewrite the problem as follows:

$$
\begin{array}{cl}
(\mathrm{PA} 2): \underset{\left\{\mathbf{w}_{k}\right\}}{\operatorname{maximize}} & \sum_{k=1}^{K} u_{k} \log _{2}\left(1+c_{o} \mathbf{h}_{k}^{T} \mathbf{w}_{k}\right) \\
\text { s.t. } & \mathbf{h}_{j}^{T} \mathbf{w}_{k}=0, \forall j \neq k \\
& \sum_{k=1}^{K}\left|\boldsymbol{j}_{l}^{T} \mathbf{w}_{k}\right| \leq P, \forall l \\
& \mathbf{h}_{k}^{T} \mathbf{w}_{k} \geq 0, \quad \forall k .
\end{array}
$$

Let $\mathbf{G}_{k} \triangleq\left[\widetilde{\mathbf{h}}_{1}, \ldots, \widetilde{\mathbf{h}}_{k-1}, \widetilde{\mathbf{h}}_{k+1}, \ldots, \widetilde{\mathbf{h}}_{\mathrm{K}}\right]^{T} \in \mathbb{R}^{(K-1) \times L}$ and its Singular Value Decomposition (SVD), $\mathbf{G}_{k}=\mathbf{U}_{k} \boldsymbol{\Sigma}_{k} \mathbf{V}_{k}^{T}$ where $\mathbf{U}_{k} \in \mathbb{R}^{(K-1) \times(K-1)}, \boldsymbol{\Sigma}_{k} \in \mathbb{R}^{(K-1) \times(K-1)}$ is a positive diagonal matrix and $\mathbf{V}_{k} \in \mathbb{R}^{L \times L}$. Lets now define the projection matrix $\mathbf{F}_{k}=\mathbf{I}-\mathbf{V}_{k} \mathbf{V}_{k}^{T}$ that can be further expressed as

$$
\mathbf{F}_{k}=\widetilde{\mathbf{V}}_{k} \widetilde{\mathbf{V}}_{k}^{T} .
$$

The matrix $\widetilde{\mathbf{V}}_{k} \in \mathbb{R}^{L \mathrm{x}(L-K+1)}$ contains the vectors of the subspace orthogonal to the interference and it satisfies that $\mathbf{V}_{k}^{T} \widetilde{\mathbf{V}}_{k}=\mathbf{0}$ and $\widetilde{\mathbf{V}}_{k}^{T} \widetilde{\mathbf{V}}_{k}=\mathbf{I}$. If we force the precoding vector, $\mathbf{w}_{k} \in \mathbb{R}^{L \times 1}$, to be a linear combination of the orthogonal vectors to the interference, i.e. $\mathbf{w}_{k}=\widetilde{\mathbf{V}}_{k} \mathbf{q}_{k}$ where $\mathbf{q}_{k} \in \mathbb{R}^{(L-K+1) \mathrm{x} 1}$, we can remove the ZF constraint since:

$$
\mathbf{G}_{k} \mathbf{w}_{k}=\left(\mathbf{U}_{k} \boldsymbol{\Sigma}_{k} \mathbf{V}_{k}^{T}\right) \tilde{\mathbf{V}}_{k} \mathbf{q}_{k}=\mathbf{0} .
$$

Therefore, we can reformulate (PA2) as follows:

$$
\begin{aligned}
\text { (PA3) : } \underset{\left\{\mathbf{q}_{k}\right\}}{\operatorname{maximize}} & \sum_{k=1}^{K} u_{k} \log _{2}\left(1+c_{o} \mathbf{h}_{k}^{T} \widetilde{\mathbf{V}}_{k} \mathbf{q}_{k}\right) \\
\text { s.t. } & \sum_{k=1}^{K}\left|\boldsymbol{j}_{l}^{T} \widetilde{\mathbf{V}}_{k} \mathbf{q}_{k}\right| \leq P, \forall l \\
& \mathbf{h}_{k}^{T} \widetilde{\mathbf{V}}_{k} \mathbf{q}_{k} \geq 0, \quad \forall k .
\end{aligned}
$$

Problem (PA3) is a concave problem and it can be solved numerically using standard optimization tools with an affordable computational complexity while guaranteeing that the optimum solution is found, along with the values of the variables (i.e. optimal values for each precoder) achieving the maximum of the cost function. In particular, we could use Lagrange duality method [14] to solve the problem.

\section{B. Suboptimal weighted sum-rate zero forcing precoding}

Coming back to (16), we can observe that if $M \ggg 1$, $P_{s, \text { elec }}(14)$ can be approximated by $1 / 3$. Thus, the BER bound can be approximated by

$$
\operatorname{BER}_{\text {MPAM }}(\mathrm{SNR}) \leq 0.1 \exp \left[-\frac{0.5 \gamma^{2}\left|\mathbf{h}_{k}^{T} \mathbf{w}_{k}\right|^{2}}{\sigma_{k}^{2}\left(M^{2}-1\right)}\right] .
$$

Denoting $\mathbf{S}_{k} \triangleq \mathbf{w}_{k} \mathbf{w}_{k}^{T}$ and considering $M=2^{r}$, (23) leads to the following rate lower-bound:

$$
r \geq \frac{1}{2} \log _{2}\left(1+c_{s o} \mathbf{h}_{k}^{T} \mathbf{S}_{k} \mathbf{h}_{k}\right) .
$$

where $c_{s o}=\frac{1}{2} \frac{\gamma^{2}}{\sigma^{2} \log \left(\frac{0.1}{\mathrm{BER}_{\mathrm{MPAM}}}\right)}$.

We will focus now on finding the optimal set of $\mathbf{S}_{k}$ that maximizes the sum-rate while satisfying the set of per-LED constraints given by (20). However, the constraints in (20) are expressed in terms of $w_{k, l}$ (optical domain) and now we need to expressed them in terms of $\mathbf{S}_{k}$ (i.e. in the electrical domain). Applying the Cauchy-Schwarz inequality to (20), we can obtain a sufficient condition in the electrical domain (25) that ensures the fulfillment of the restriction (20):

$$
\sum_{k=1}^{K}\left|w_{l, k}\right|^{2} \leq \frac{P^{2}}{K} .
$$

This constraint is equivalent to:

$$
\sum_{k=1}^{K} \operatorname{Tr}\left(\mathbf{B}_{l} \mathbf{S}_{k}\right) \leq \frac{P^{2}}{K}, \quad l=1, \ldots, L
$$

where $\mathbf{B}_{l} \triangleq \operatorname{diag}(0, \ldots, 1,0, \ldots 0)$ is a diagonal matrix, with a 1 in the $l$-th element of the diagonal and the rest elements of the diagonal are zero.

The approach followed so far, i.e. expressing the costfunction in terms of $\mathbf{S}_{k}$ and obtaining (25) is actually similar to the approach followed in [6] to find the optimal precoders. Note, however, that (25) is a sufficient condition but not a necessary condition. This means, that we could relax the 
bound, and still find better solution that it is still feasible (i.e. a better solution that still fulfills the optical power constraint).

On the other hand, we can find a necessary condition. By applying the norm property $\|\cdot\|_{2} \leq\|\cdot\|_{1}$, we can affirm that $\forall l$,

$$
\sqrt{\sum_{k=1}^{K}\left|w_{l, k}\right|^{2}} \leq \sum_{k=1}^{K}\left|w_{l, k}\right| \leq P
$$

and, thus,

$$
\sum_{k=1}^{K}\left|w_{l, k}\right|^{2} \leq P^{2} .
$$

Therefore, the squared norm of the optimal weight vector should be within the interval $\left[\frac{P^{2}}{K}, P^{2}\right]$. Note that any vector whose $\sum_{k=1}^{K}\left|w_{l, k}\right|^{2}>P^{2}$ will be not feasible (the optical power constraint (20) will not be met). On the other hand, for any vector whose $\sum_{k=1}^{K}\left|w_{l, k}\right|^{2}<\frac{P^{2}}{K}$, we can find another vector that fulfills (20) and provides a higher rate. For the time being, we assume that $p \in\left[\frac{P^{2}}{K}, P^{2}\right]$ meets the power requirement $\forall l$.

Regarding the ZF constraint, in order to eliminate the interuser interference, the weights should satisfy that $\mathbf{h}_{j}^{T} \mathbf{w}_{k}=$ $0, \forall j \neq k$. This ZF constraint is equivalent to $\mathbf{h}_{j}^{T} \mathbf{S}_{k} \mathbf{h}_{j}=$ $0, \forall j \neq k$.

Finally, we can formulate our problem as

$$
\begin{array}{cl}
(\mathrm{PB} 1): \underset{\left\{\mathbf{S}_{k}\right\}}{\operatorname{maximize}} & \frac{1}{2} \sum_{k=1}^{K} u_{k} \log _{2}\left(1+c_{s o} \mathbf{h}_{k}^{T} \mathbf{S}_{k} \mathbf{h}_{k}\right) \\
\text { s.t. } & \mathbf{h}_{j}^{T} \mathbf{S}_{k} \mathbf{h}_{j}=0, \forall j \neq k \\
& \sum_{k=1}^{K} \operatorname{Tr}\left(\mathbf{B}_{l} \mathbf{S}_{k}\right) \leq p \quad \forall l \\
& \mathbf{S}_{k} \succeq 0 \quad \forall k
\end{array}
$$

This problem is similar to the formulated in [9] in a RF framework. In order to simplify the derivations, let us include the constant $c^{\prime}$ in the channel. The resulting channel is then defined by $\widetilde{\mathbf{h}}_{k}=\sqrt{c_{s o}} \mathbf{h}_{k}$.

Using the projection matrix defined in (21), $\mathbf{F}_{k}=\widetilde{\mathbf{V}}_{k} \widetilde{\mathbf{V}}_{k}^{T}$, we can state that the optimal solution of (PB1) is given by $\mathbf{S}_{k}=\widetilde{\mathbf{V}}_{k} \mathbf{Q}_{k} \widetilde{\mathbf{V}}_{k}^{T} \quad \forall k$ where $\mathbf{Q}_{k} \in \mathbb{R}^{(L-K+1) \mathrm{x}(L-K+1)}$. The structure for $\mathbf{S}_{k}$ ensures the $\mathrm{ZF}$ condition since the matrix $\widetilde{\mathbf{V}}_{k} \in \mathbb{R}^{L \mathrm{x}(L-K+1)}$ contains the vectors of the subspace orthogonal to the interference and, consequently, $\mathbf{V}_{k}^{T} \widetilde{\mathbf{V}}_{k}=\mathbf{0}$ that implies that $\widetilde{\mathbf{h}}_{j}^{T} \mathbf{S}_{k} \widetilde{\mathbf{h}}_{j}=0, \forall j \neq k$. Therefore, the problem (PB1) can be written as

$$
\begin{aligned}
& \text { (PB2) : } \underset{\left\{\mathbf{Q}_{1}, \ldots, \mathbf{Q}_{K}\right\}}{\operatorname{maximize}} \frac{1}{2} \sum_{k=1}^{K} u_{k} \log _{2}\left(1+\widetilde{\mathbf{h}}_{k}^{T} \widetilde{\mathbf{V}}_{k} \mathbf{Q}_{k} \widetilde{\mathbf{V}}_{k}^{T} \widetilde{\mathbf{h}}_{k}\right) \\
& \text { s.t. } \quad \sum_{k=1}^{K} \operatorname{Tr}\left(\mathbf{B}_{l} \widetilde{\mathbf{V}}_{k} \mathbf{Q}_{k} \tilde{\mathbf{V}}_{k}^{T}\right) \leq p, \quad \forall l \\
& \mathbf{Q}_{k} \succeq \mathbf{0}, \quad \forall k \text {. }
\end{aligned}
$$

This problem is concave and again it can be solved with affordable complexity. In particular, we could use the Lagrange duality method [14], that iteratively computes the primal and dual variables. The dual variables, i.e. the set of non-negative multipliers associated with the power constraint for each LED, $\left\{\mu_{l}\right\}$, can be obtained through the ellipsoid method [15].

Applying the Lagrange duality method is particularly interesting now, since, for a given the set of non-negative multipliers $\left\{\mu_{l}\right\}$, the optimum values of $\mathbf{w}_{k}^{*}$ can be obtained in closed form as follows:

$$
\mathbf{w}_{k}^{*}=\lambda_{k}^{1 / 2} \widetilde{\mathbf{V}}_{k}\left(\widetilde{\mathbf{V}}_{k}^{T} \mathbf{B}_{\mu} \widetilde{\mathbf{V}}_{k}\right)^{-1 / 2} \hat{\boldsymbol{v}}_{k}
$$

where $\mathbf{B}_{\mu}=\sum_{l=1}^{L} \mu_{l} \mathbf{B}_{l}$ and $\hat{\boldsymbol{v}}_{k}^{T} \in \mathbb{R}^{(L-K+1) \times 1}$ is obtained through the SVD of

$$
\mathbf{h}_{k}^{T} \widetilde{\mathbf{V}}_{k}\left(\widetilde{\mathbf{V}}_{k}^{T} \mathbf{B}_{\mu} \tilde{\mathbf{V}}_{k}\right)^{-1 / 2}=\hat{\sigma_{k}} \hat{\boldsymbol{v}}_{k}^{T}
$$

with $\hat{\sigma_{k}}>0$.

The value of $\lambda_{k}$ is given by the water-filling algorithm:

$$
\lambda_{k}=\left(\frac{u_{k}}{2 \log 2}-\frac{1}{\hat{\sigma}_{k}^{2}}\right)^{+} .
$$

Proof: Please refer to [9].

As discussed previously, setting $p=\frac{P^{2}}{K}$ we can ensure that the solution obtained fulfills the optical power nonnegative constraint. Note that by increasing the value of $p$ we make the problem (PB1) less restricted and therefore we can obtain a better solution. Because of this, we propose to use the bisection method [16] to find the highest value of $p$ that it is low enough to fulfill all the optical power nonnegative constraints. Initially, we take the interval given by $\left[\frac{P^{2}}{K}, P^{2}\right] \forall l$ and solve the problem considering $p$ equal to the middle point of the interval. If the found solution verify the optical constraint $\forall l$, the search interval will be updated to $\left[\frac{P^{2}+P^{2} / K}{2}, P^{2}\right]$. Otherwise, the search interval will be updated as $\left[\frac{P^{2}}{K}, \frac{P^{2}+P^{2} / K}{2}\right]$. The procedure is repeated until the convergence to the optimum value of $p$ (i.e. the highest value of $p$ that is feasible) with a resolution smaller than a given percentage of the search interval length $P^{2}-P^{2} / K$. Note that the procedure converges always with exponential speed. For instance, for a resolution of $1 \%$ only seven iterations are required.

\section{Zero forcing precoding with pseudo-inverse}

A much simple precoder can be obtained through the use of the pseudo-inverse. Taking the definition of the pseudoinverse, it is easy to realize that $\mathbf{W}=m \mathbf{H}^{\dagger}=m \widetilde{\mathbf{W}}$ generates multiple independent sub-channels since

$$
\mathbf{y}=\gamma \mathbf{H} \mathbf{x}+\mathbf{n}=\gamma \mathbf{H}\left(m \mathbf{H}^{\dagger} \mathbf{s}+\mathbf{b}\right)+\mathbf{n}=m \gamma \mathbf{s}+\gamma \mathbf{H b}+\mathbf{n}
$$

where $m$ is a scale factor of the precoding matrix.

The inequalities (9) and (11) define a possible set of values for $m$ and $\mathbf{b}$ that satisfy both constraints. For the LED $l$ th, the selected pair of $m$ and $b_{l}$ is the one that achieves the highest SNR. It can be easily proved that the maximum SNR coincides with the intersection between (9) and (11) that leads to the optimum value of $b_{l}^{*}=\frac{P_{o p t_{\max }}}{2}$. Thus, we only 
have two design parameters, $m$ and $b$, that need to, on the one hand, maximize the SNR and, on the other, meet all the requirements of the different LEDs. Substituting the value of $b_{l}^{*}$ in (9) and (11), a unique constraint has to be satisfied:

$$
\sum_{k=1}^{K}\left|\widetilde{w}_{l, k}\right| \leq \frac{P_{o p t_{\max }}}{2 m}, \quad l=1, \ldots, L .
$$

This inequality is satisfied when the most restrictive case is considered, i.e. when the right side of the inequation (33) is maximal, leading to $m=\frac{P_{o p t_{\max } / 2}}{\max _{l}\left\{\sum_{k=1}^{K}\left|\widetilde{w}_{l, k}\right|\right\}}$.

\section{NumERICAL RESUlts}

In this section, we examine an adaptive modulation scheme where the data rate, transmitted power and constellation size are adjusted to obtain an uncoded BER under $10^{-6}$ subject to the non-negativity and optical power constraints. The objective of the adaptive modulation is a real-time balancing of the constellation size in order to always achieving a BER under the target. The adaptive scheme takes advantage of the time-varying nature of the channel to obtain the modulation which allows the highest rate for a given link quality. Furthermore, it allows to transmit at high speed under favorable channel conditions and reduce the rate when the conditions are degraded.

Using equation (16) we have been able to obtain the BER achieved by each user from its SNR when using different constellation sizes. In each case, the selected $M$ is the highest one that ensures a BER under the target. In Table II, the rate is specified for the three different proposed schemes using the values and the distribution specified in Table I and Figure 2. For the purpose of simplification, we have assumed that the rate's weights $u_{k}$ are 1 .

Table I

SIMULATION PARAMETERS

\begin{tabular}{|c|c|c|}
\hline \multicolumn{3}{|l|}{ LED parameters } \\
\hline Number of LEDs & $L$ & 12 \\
\hline Maximum transmitted optical power & $P_{o p t_{\max }}$ & $20 \mathrm{~W}$ \\
\hline Semi-angle at half power & $\Phi_{1 / 2}$ & $70^{\circ}$ \\
\hline Center luminous intensity & $I_{0}$ & $0.73 \mathrm{~cd}$ \\
\hline \multicolumn{3}{|l|}{ PD parameters } \\
\hline Number of users & $K$ & 6 \\
\hline Field of View & FOV & $60^{\circ}$ \\
\hline Detector physical area of a PD & $A_{r}$ & $1 \mathrm{~cm}^{2}$ \\
\hline Gain of the optical filter & $T_{s}(\alpha)$ & 1 \\
\hline Refractive index of the concentrator & $\kappa$ & 1.5 \\
\hline Responsivity & $\gamma$ & $0.53 \mathrm{~A} / \mathrm{W}$ \\
\hline Noise parameters & ters can & found in [11] \\
\hline
\end{tabular}

For the pseudo-inverse precoder, the maximum modulation that achieves the target BER is 8-PAM for all the users. Consequently, the achieved rate is $r=\log _{2} 8=3[\mathrm{bps} / \mathrm{Hz}]$. Of course, if we took MPAM constellations with less symbols ( $M=2$ or $M=4$ ) we would also get a BER under the target although a lower rate would be achieved. It is worth noting that the achieved users' rates are the same since all the users have a similar BER and SNR for all $M$. However, the shot noise depends on the average optical power received that is different for each user since it depends on the DC component,

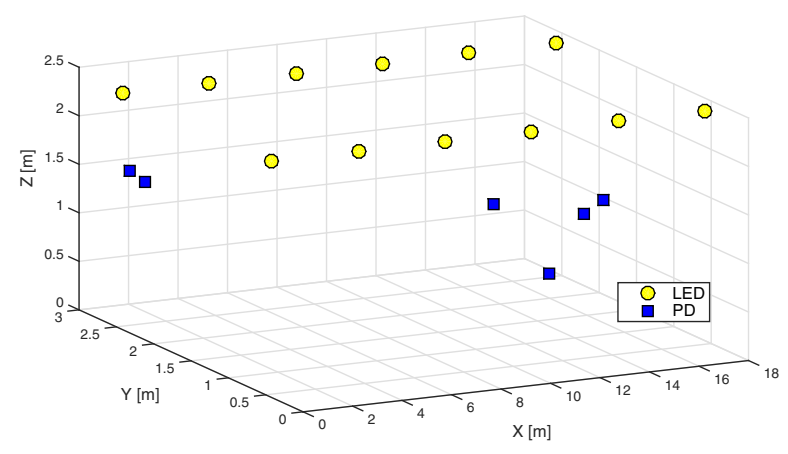

Figure 2. LEDs and PDs distribution.

Hb. When using the optimal and suboptimal precoders, the users have very different SNRs and thus the users' rates are different (see Table II). In this specific case, the optimal and suboptimal designs achieve the same rates (see Table II). Firstly, the higher constellation sizes are considered. With $M=64$ two users with the optimal and the suboptimal precoder achieve a BER under the target and with $M=16$, another user with the optimal $\mathrm{ZF}$ and the suboptimal $\mathrm{ZF}$ precoder gets a BER under the objective. With $M=8$ and $M=4$, the rest of the users satisfy the BER requirement with the two schemes.

In addition to these ZF schemes, a TDMA system has also been taken into consideration. TDMA achieves the lowest BER with higher $M$, because in each transmission all power is devoted to one user. Nevertheless, each user transmits only $1 / K$ of the time. Therefore, the effective rates are reduced by a factor $K$ and this makes it the least spectrally efficient. In Table II, the effective users' rates with TDMA have been obtained dividing the instantaneous rates by the number of slots, i.e. by $K=6$.

Table II

RATE ACHIEVED BY EACH USER [BPS/Hz]

\begin{tabular}{|l|c|c|c|c|c|c|}
\hline User & $\mathbf{1}$ & $\mathbf{2}$ & $\mathbf{3}$ & $\mathbf{4}$ & $\mathbf{5}$ & $\mathbf{6}$ \\
\hline Optimal precoder & 2 & 2 & 3 & 4 & 6 & 6 \\
Suboptimal precoder & 2 & 2 & 3 & 4 & 6 & 6 \\
Pseudo-inverse & 3 & 3 & 3 & 3 & 3 & 3 \\
TDMA & 0.83 & 0.83 & 0.83 & 0.83 & 1 & 1.17 \\
\hline
\end{tabular}

The results in Table II are for a particular set of channel gains. In order to evaluate the performance and capture the randomness of the channel, multiple realizations have been run. Each realization will satisfy the BER requirement through the variation of the MPAM constellation. To illustrate the results, the rate's Cumulative Distribution Function (CDF) has been computed in Figure 3.

Results from Figure 3 show that, by far, the worst scheme is TDMA in terms of rate. For the worst $30 \%$ of the users, the pseudo-inverse achieves more than twice the rate of TDMA, while the other schemes triplicate the rate of the TDMA approach. The suboptimal precoder, despite being an approximated solution, presents a very similar performance than the optimal precoder. On the other hand, the compu- 


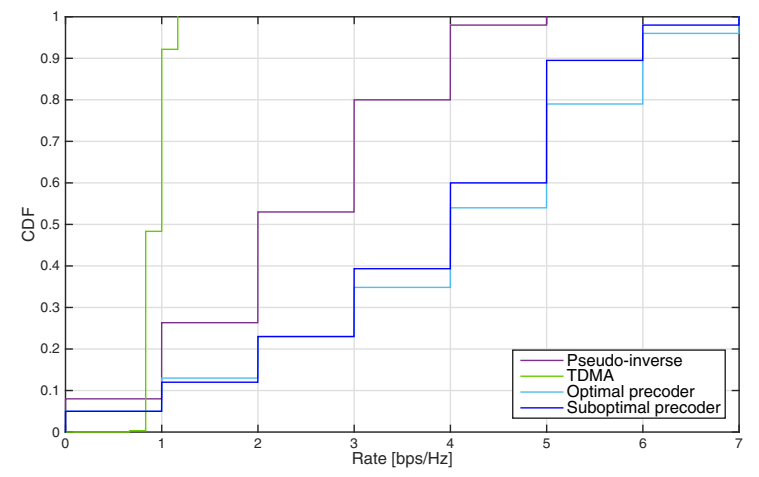

Figure 3. Rate CDF for the different MISO precoding schemes.

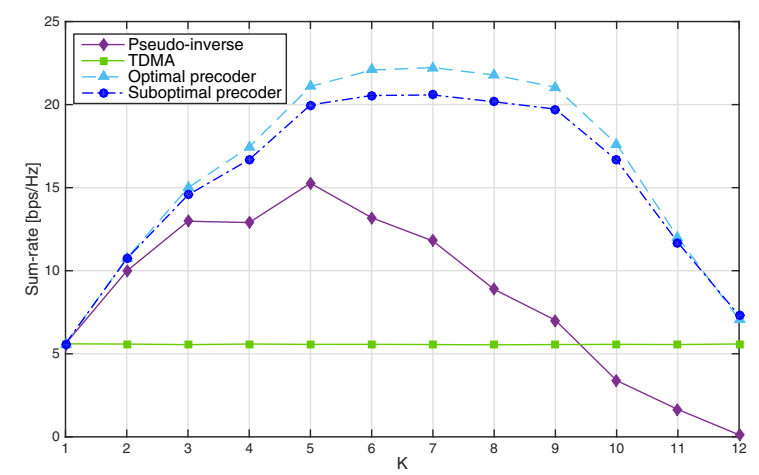

Figure 4. Sum-rate achieved increasing the number of users $K$ for a deployment of 12 LEDs.

tational time required to compute the optimal precoder is higher. Specifically, using the available optimization functions of MATLAB to solve problem (PA3) requires 30\% more time than computing the sub-optimal precoder as described in section III-B (considering a resolution of $1 \%$ for the bisection procedure).

In Figure 4, the average sum-rate is displayed for different number of users $K$ for the LED's distribution specified in Figure 2. Several realizations have been carried out by randomly generating the users' positions. It is worth noting that the sum-rate does not steadily increase with the number of users due to three effects: first, the available optical power per user; second, the difficulties in forcing spatial zeros for an increased number of terminals in a limited area; and third, when obtaining the inequalities (9) and (11) from (8) and (10), respectively, we have considered the worst case, i.e. that all the symbols are such that the contributions of all users to the per-LED constraint are added constructively (hence the absolute value). Therefore, increasing the number of users, will constraint more the value $\left|w_{l, k}\right|$ for each specific user.

Figure 4 provides some design guidelines about the maximum number of terminals to be used, and show that the proposed optimum approach outperforms the alternate precoding schemes. The suboptimal precoder presents poorer results in comparison with the optimal precoder but implies a remarkable improvement against the pseudo-inverse precoding and single-user TDMA approaches. Note that TDMA always reaches the same sum-rate, independently of the number of users, being by far the worst approach.

\section{COnClusions}

This paper has tackled the design of coordinated joint transmission techniques for a MISO multipoint to multipoint setup for VLC. Two critical constraints are included: the nonnegativity for the VLC signal imposed by IM/DD and the maximum optical power transmitted per LED.

The two schemes proposed are obtained from the resolution of an optimization problem that considers as figure of merit the weighted sum-rate for a MPAM constellation. After proper manipulation, both could be solved by applying convex optimization techniques and has lead to two different precoders. For the first precoder, a concave non-differentiable problem has been solved numerically. The second precoder is derived through an approximation of the initial problem and an almost closed-form for the suboptimal precoding matrix has been found. Both schemes stand out significantly from TDMA and the pseudo-inverse precoding schemes, both in terms of sum-rate and outage rate.

\section{REFERENCES}

[1] S. Dimitrov and H. Haas, Principles of LED Light Communications. Torwards Networked Li-Fi. Cambridge University Press, 2015.

[2] A. Paulraj, R. Nabar, and D. Gore, Introduction to Space-time Communications. Cambridge University Press, 2003.

[3] J. M. Kahn and J. R. Barry, "Wireless Infrared Communication," Proceedings of the IEEE, vol. 85, no. 2, 1997.

[4] H. Yang, J. Chen, Z. Wang, and C. Yu, "Performance of a Precoding MIMO System for Decentralized Multiuser Indoor Visible Light Communications," IEEE Photonics Journal, vol. 5, no. 4, 2014.

[5] H. Shen, Y. Deng, W. Xu, and C. Zhao, "Rate-Maximized Zero-Forcing Beamforming for VLC Multiuser MISO Downlinks," IEEE Photonics Journal, vol. 8, no. 1, February 2016.

[6] T. V. Pham, H. L. Minh, Z. Ghassemlooy, T. Hayashi, and A. T. Pham, "Sum-Rate Maximization of Multi-User MIMO Visible Light Communications," in IEEE ICC, Workshop on Visible Light Communications and Networking (VLCN), 2015.

[7] Z. Yu, R. J. Baxley, and G. T. Zhou, "Multi-User MISO Broadcasting for indoor Visible Light Communication," in IEEE International Conference on Acoustics, Speech and Signal Processing, 2013.

[8] A. Wiesel, Y. C. Eldar, and S. Shamai, "Zero-Forcing Precoding and Generalized Inverses," IEEE Transactions on Signal Processing, vol. 56, no. 9, September 2008.

[9] R. Zhang, "Cooperative Multi-Cell Block Diagonalization with PerBase-Station Power Constraints," IEEE Journal on Selected Areas in Communications, vol. 28, no. 9, December 2010.

[10] P. H. Pathak, X. Feng, P. Hu, and P. Mohapatra, "Visible Light Communication, Networking, and Sensing: A Survey, Potential and Challenges," IEEE Communications Surveys and Tutorials, vol. 17, no. 4, 2015.

[11] T. Komine and M. Nakagawa, "Fundamental analysis for visiblelight communication system using LED lights," IEEE Transactions on Consumer Electronics, vol. 50, no. 1, February 2004.

[12] A. Vavoulas, H. G. Sandalidis, T. A. Tsiftsis, and N. Vaiopoulos, "Coverage Aspects of Indoor VLC Networks," Journal of Lightwave Technology, vol. 33, no. 23, December 2015.

[13] S. T. Chung, S. Vishwanath, and A. Goldsmith, "Degrees of Freedom in Adaptive Modulation: A unified view," IEEE Transactions on Communications, vol. 49, no. 9, September 2001.

[14] S. Boyd and L. Vandenberghe, Convex Optimization. Cambridge University Press, 2004.

[15] R. G. Bland, D. Goldfarb, and M. J. Todd, "The Ellipsoid Method: a Survey," Operations Research, vol. 29, no. 6, November-December 1981.

[16] R. L. Burden and J. D. Faires, Numerical analysis, 3rd ed. Prindle, Weber and Schmidt, 1985. 